\title{
MODULES OVER DEDEKIND RINGS AND VALUATION RINGS
}

\author{
BY \\ IRVING KAPLANSKY
}

1. Introduction. This paper has two purposes. In $\$ 4$ our objective is to push forward the theory of modules over Dedekind rings to approximately the same point as the known theory of modules over principal ideal rings. In $\$ 5$ we prove several results concerning modules over valuation rings. In Theorem 12 we give a complete structure theorem for torsion-free modules of countable rank over a maximal valuation ring. In Theorem 14 we show that any finitely generated module over an almost-maximal valuation ring is a direct sum of cyclic modules; this provides a simpler proof and generalization of Theorem 11.1 in [6] $\left(^{1}\right)$.

2. Basic notions. Let $R$ be an integral domain (commutative ring with unit and no divisors of 0 ). In saying that $M$ is an $R$-module, we shall always mean that the unit element of $R$ acts as unit operator on $M$. If $x$ is any element in $M$, the set of $\alpha$ in $R$ with $\alpha x=0$ is called the order ideal of $x$. If all elements of $M$ have a nonzero order ideal, we call $M$ a torsion module. If all nonzero elements of $M$ have 0 as their order ideal, we say that $M$ is torsionfree. In general, the elements of $M$ with a nonzero order ideal form a submodule $T$ of $M$ called the torsion submodule, and the quotient module $M / T$ is torsion-free.

In case $M$ is a torsion-free $R$-module, we may speak unambiguously of the maximum number of linearly independent elements in $M$, and we call this cardinal number the rank of $M$. There is another point of view that is useful. We may extend the coefficient domain of $M$ from $R$ to its quotient field $K$; that is, we may form the Kronecker product $K \times M$ of the $R$-modules $K$ and $M$. Then $K \times M$ may be regarded as a vector space over $K$, and its dimension coincides with the rank of $M$.

We shall not attempt the delicate task of assigning a meaning to "rank" in the case of torsion modules.

If $M$ is a torsion-free $R$-module of rank one, it is easy to see that $M$ is isomorphic to an (integral or fractional) ideal $I$ in $R$. (In this connection, no demand should be made concerning the boundedness of the denominators occurring in $I$; such boundedness is equivalent to the possibility of selecting $I$ to be an integral ideal.)

We next introduce Prüfer's [10] concept of "Servanzuntergruppe," but we prefer to use Braconnier's terminology [3]. The submodule $S$ of the

Received by the editors July 30,1951 .

(1) Numbers in brackets refer to the bibliography at the end of the paper. 
$R$-module $M$ is said to be pure if $\alpha S=S \cap \alpha M$ for every $\alpha$ in $R$.

The reader may find the following remarks helpful. They are easily proved and will be used without further comment. (a) Any direct summand is pure. (b) A pure submodule of a pure submodule is pure. (c) The torsion submodule is pure. (d) If $M / S$ is torsion-free, then $S$ is pure. (e) If $M$ is torsion-free, then the intersection of any set of pure submodules of $M$ is again pure. Hence any subset of $M$ is contained in a unique smallest pure submodule.

Numerous examples show that a pure submodule need not be a direct summand. However in various special cases we shall be able to prove that this converse does hold.

Finally we define an $R$-module $M$ to be divisible if $\alpha M=M$ for all nonzero $\alpha$ in $R$. We note that if $M$ is divisible, then a submodule of $M$ is divisible if and only if it is pure; also if $M$ is any $R$-module, then any divisible submodule of $M$ is pure (by a divisible submodule we mean a submodule which is divisible as a module in its own right). A torsion-free divisible $R$-module is evidently the same thing as a vector space over the quotient field of $R$.

3. Some lemmas. Before proceeding to special classes of rings, we shall prove three lemmas that are valid for an arbitrary integral domain. The first concerns the question of the uniqueness of the representation of a torsionfree module as a direct sum of modules of rank one. If $I$ and $J$ are (integral or fractional) ideals in $R$, one knows that they are isomorphic $R$-modules if and only if there exists a nonzero $\alpha$ in the quotient field of $R$, with $I=\alpha J$. Following the usual terminology, we then say that $I$ and $J$ are in the same class. We now show that for the direct sum of a finite number of ideals, the class of the product is an invariant. In general there are other invariants, but in a particular case (Theorem 2) we shall find it to be the sole invariant.

Lemma 1. Let $R$ be an integral domain, and let $I_{1}, \cdots, I_{m}, J_{1}, \cdots, J_{m}$ be (integral or fractional) ideals in $R$ such that $I_{1} \oplus \cdots \oplus I_{m}$ and $J_{1} \oplus \cdots \oplus J_{m}$ are isomorphic $R$-modules. Then $I_{1} I_{2} \cdots I_{m}$ and $J_{1} J_{2} \cdots J_{m}$ are in the same class.

Proof. It is convenient to assume (as we can without loss of generality) that each of the ideals contains $R$. In the isomorphism between the two direct sums, suppose the element 1 in $I_{r}$ corresponds to $\left(\alpha_{r 1}, \cdots, \alpha_{r m}\right)$, where $\alpha_{r s} \in J_{s}$. We then find

$$
J_{s}=\alpha_{18} I_{1}+\cdots+\alpha_{m s} I_{m} \quad(s=1, \cdots, m) .
$$

Let $\gamma$ be the determinant $\left|\alpha_{r s}\right|$, and let $\delta$ be a typical term in the expansion of this determinant. On multiplying the equations (1), we express $J_{1} \cdots J_{m}$ as a sum of ideals, among which we find all $\delta I_{1} \cdots I_{m}$. Hence

$$
J_{1} \cdots J_{m} \supset \gamma I_{1} \cdots I_{m}
$$


We now invert the procedure. Suppose the element 1 in $J_{r}$ corresponds to $\left(\beta_{r 1}, \cdots, \beta_{r m}\right)$, where $\beta_{r s} \in J_{s}$. Then the matrix $\left(\beta_{r s}\right)$ is the inverse of the matrix $\left(\alpha_{r s}\right)$. We thus obtain the inclusion

$$
I_{1} \cdots I_{m} \supset \gamma^{-1} J_{1} \cdots J_{m}
$$

and this proves the lemma.

The next lemma could easily be stated and proved in more general contexts. (By the term "direct sum" we mean the "weak" direct sum, where only a finite number of nonzero components are allowed in each element.)

Lemma 2. Let $M$ be any module, $S$ a submodule, $M / S$ a direct sum of modules $U_{i}$, and $T_{i}$ the inverse image in $M$ of $U_{i}$. Suppose $S$ is a direct summand of each $T_{i}$. Then $S$ is a direct summand of $M$.

Proof. Let $W_{i}$ be a complementary summand of $S$ in $T_{i}$, and $W$ the union of $\left\{W_{i}\right\}$. Then $W$ maps onto all of $M / S$, whence $S+W=M$. Again suppose $x=\sum y_{i}$ is in $S \cap W$, where $y_{i} \in W_{i}$. Then on passing to $M / S$ we see that each $y_{i}$ is in $S, y_{i}=0, x=0$. Thus $S \cap W=0$ and $M=S \oplus W$.

Let $R$ be any integral domain with quotient field $K$, and $I$ an (integral or fractional) ideal in $R$. We recall that $I^{-1}$ is defined as the set of all $\alpha$ in $K$ with $\alpha I \subset R ; I$ is said to be invertible if $I I^{-1}=R$. Any invertible ideal is finitely generated.

Lemma 3. Let $R$ be an integral domain, $M$ an $R$-module, $S$ a submodule such that $M / S$ is a direct sum of modules which are isomorphic to invertible ideals in $R$. Then $S$ is a direct summand of $M$.

Proof. Lemma 2 reduces the problem to the case where $M / S$ is itself isomorphic to an invertible ideal $I$. We therefore make this assumption, and we furthermore make the provisional assumption that $M$ is torsion-free.

Since $I I^{-1}=R$, there exist elements $\alpha_{i}, \beta_{i}$ in $I$ and $I^{-1}$ such that $\sum \alpha_{i} \beta_{i}$ $=1$. Let $y_{i}$ be the elements in $M / S$ which correspond to $\alpha_{i}$, let $\gamma$ be any nonzero element in $I$, let $x_{i}$ be any elements in $M$ mapping on $y_{i}$, and set $z=\gamma \sum \beta_{i} x_{i}$. (Notice that $\gamma \beta_{i} \in R$, so that $z$ is a well defined element of $M$.) Let $T$ be the pure submodule generated by $z$; since $M$ is torsion-free, this is a well defined submodule of rank one. We claim that $T$ is the desired complementary summand of $S$. That $S \cap T=0$ is clear; we need only show $S+T$ $=M$, that is, we must show that $T$ maps on all of $M / S$. For this it suffices to prove that $y_{i}$ is in the image of $T$ (note that the elements $\alpha_{i}$ generate $I$, and so the elements $y_{i}$ generate $\left.M / S\right)$. Now $\alpha_{i} \beta_{j} \in R$. Hence $t=\alpha_{i} \gamma^{-1} z$ $=\alpha_{i} \sum \beta_{j} x_{j}$ is in $M$, and consequently $t$ is in $T$. The image of $t$ is $\alpha_{i} \sum \beta_{j} y_{j}$. In the isomorphism of $M / S$ and $I, \sum \beta_{j} y_{j}$ corresponds to $\sum \beta_{j} \alpha_{j}=1$. Hence $\alpha_{i} \sum \beta_{j} y_{j}=y_{i}$, as desired.

We pass to the general case where $M$ is no longer assumed to be torsionfree. Select a homomorphism $\phi$ of a free $R$-module $F$ onto $M$. This gives rise 
to a homomorphism from $F$ onto $M / S$, say with kernel $G$ ( $G$ is also the inverse image of $S$ under $\phi)$. By what we have just proved, $G$ is a direct summand of $F$ : say $F=G \oplus H$. Let $V=\phi(H)$. Manifestly $S+V=M$. Again, since the kernel of $\phi$ is contained in $G, \phi(G)$ and $\phi(H)$ have only 0 in common. Thus $S \cap V=0$ and $M=S \oplus V$. This concludes the proof of Lemma 3.

4. Dedekind rings. A Dedekind ring is an integral domain in which classical ideal theory holds: that is, every ideal is uniquely a product of prime ideals or alternatively, every ideal is invertible. For our first two theorems we find it possible to consider the more general class of integral domains in which it is assumed only that every finitely generated ideal is invertible.

THEOREM 1. Let $R$ be an integral domain in which every finitely generated ideal is invertible, and let $M$ be a finitely generated $R$-module with torsion submodule $T$. Then $T$ is is a direct summand of $M$, and $M / T$ is a direct sum of modules of rank one (necessarily isomorphic to invertible ideals).

Proof. That $T$ is a direct summand of $M$ will follow from Lemma 3, as soon as we have proved the last statement of the theorem. So let us suppose that $M$ is actually a torsion-free finitely generated $R$-module. Take any nonzero element in $M$ and form the pure submodule $S$ of rank one that it generates. Then $M / S$ is again torsion-free and finitely generated. Its rank is one less than the rank of $M$. By induction on the rank, we may assume that $M / S$ is a direct sum of modules of rank one. Lemma 3 now applies to show that $M$ is the direct sum of $S$ and $M / S$, and this proves the theorem.

We turn now to the uniqueness question associated with Theorem 1. It is a classical result of Steinitz [13] that, over a Dedekind ring, the rank $n$ and the class of the product $I_{1} I_{2} \cdots I_{n}$ are a complete set of invariants for a torsion-free module $I_{1} \oplus I_{2} \oplus \cdots \oplus I_{n}$. In Theorem 2 we are able to generalize this result, provided we supplement the hypothesis that finitely generated ideals are invertible with another hypothesis known to be satisfied in a Dedekind ring $\left({ }^{2}\right)$.

We also take up this uniqueness question in the case of infinite rank. The result is typical of infinite algebra: the invariant evaporates.

THEOREM 2. Let $R$ be an integral domain which satisfies the following two conditions: every finitely generated ideal in $R$ is invertible, and if $I$ is a nonzero finitely generated ideal in $R, R / I$ is a ring in which every finitely generated ideal is principal. Then:

(a) If $M$ is a finitely generated torsion-free $R$-module, a complete set of invariants for $M$ is the rank $n$ together with the class of the product $I_{1} I_{2} \cdots I_{n}$ in a representation of $M$ as a direct sum of ideals $I_{1} \oplus \cdots \oplus I_{n}$.

(2) A suitable infinite algebraic extension of a Dedekind ring gives an example of a ring (other than a Dedekind ring) satisfying the hypotheses of Theorem 2. For an explicit example we may take the ring of all algebraic integers. 
(b) If $N$ is an R-module which is a direct sum of an infinite number of invertible ideals, then $N$ is free.

Proof. (a) That the class of $I_{1} \cdots I_{n}$ is an invariant was shown in Lemma 1 . We have to show that this class, together with the integer $n$, forms a complete set of invariants. To do this, it suffices by an evident induction to treat the case $n=2$, that is to say, we wish to show that $M=I_{1} \oplus I_{2}$ is isomorphic to $R \oplus I_{1} I_{2}$. Suppose we have shown that $M$ contains a pure submodule $S$ isomorphic to $R$. Then by Lemma $3, M$ is the direct sum of $S$ and $M / S$, and $M / S$ will necessarily be isomorphic to $I_{1} I_{2}$. So our task is reduced to finding the submodule $S$. We can of course suppose that $I_{2}$ is an integral ideal. Let $\alpha$ be any nonzero element in $I_{1}$. Then $\alpha I_{1}^{-1} I_{2}$ is a nonzero integral ideal contained in $I_{2}$. By hypothesis, $I_{2}$ maps onto a principal ideal in $R / \alpha I_{1}^{-1} I_{2}$, that is, there exists an element $\beta$ in $I_{2}$ such that $I_{2}=(\beta)+\alpha I_{1}^{-1} I_{2}$, whence

$$
\alpha I_{1}^{-1}+\beta I_{2}^{-1}=R
$$

Now consider the element $x=(\alpha, \beta)$ of $M=I_{1} \oplus I_{2}$. The pure submodule generated by $x$ is isomorphic to the ideal $K$ consisting of all $\gamma$ (in the quotient field of $R$ ) with $\gamma x \in M$; that is, all $\gamma$ with $\gamma \alpha \in I_{1}$ and $\gamma \beta \in I_{2}$. Thus $K=\alpha^{-1} I_{1}$ $\cap \beta^{-1} I_{2}$ and is the inverse of the ideal appearing on the left of (2). Hence $K=R$, as desired.

(b) If $N$ is of uncountable rank, we may break it into a direct sum of pieces of countable rank. Since it suffices to treat each of these pieces separately, we may assume that $N$ itself is of countable rank, whence it is countably generated. Let $x_{1}, x_{2}, \cdots$ be a countable set of generators for $N$.

We proceed to build a sequence of submodules $\left\{K_{j}\right\}$ of $N$ with the properties:

(1) $K_{1} \subset K_{2} \subset K_{3} \subset \cdots$,

(2) each $K_{j}$ is free,

(3) $K_{j+1}$ is the direct sum of $K_{j}$ and a free module,

(4) $N$ is the direct sum of $K_{j}$ and a module $L_{j}$ which is an infinite direct sum of finitely generated modules of rank one,

(5) the union of all the $K$ 's is $N$.

It is evident that the existence of such a sequence implies that $N$ is free.

We take $K_{1}=0$, and suppose $K_{r}$ has been selected. Let $y$ denote the first of the $x$ 's which is not in $K_{r}$, and $z$ the $L_{r}$-component of $y$ in the decomposition $N=K_{r} \oplus L_{r}$. By hypothesis, $L_{r}$ is a direct sum of modules $\left\{P_{t}\right\}$ of rank one where $P_{t}$ is isomorphic to the invertible ideal $I_{t}$ in $R$. Suppose $z$ is in $P_{1}$ $\oplus \cdots \oplus P_{s}$, and write $I=I_{1} I_{2} \cdots I_{s}$. By part (a) of the present theorem, $P_{s+1} \oplus P_{s+2}$ can be rewritten as $G \oplus H$ where $G$ and $H$ are isomorphic to $I^{-1}$ and $I I_{s+1} I_{s+2}$ respectively. Then the module $P_{1} \oplus \cdots \oplus P_{s} \oplus G$ is free, and we take $K_{r+1}$ to be the direct sum of it and $K_{r}$, while $L_{r+1}=H \oplus P_{s+3}$ $\oplus P_{s+4} \oplus \cdots$. With this choice of $K_{r+1}$ and $L_{r+1}$ we continue to satisfy 
properties (1)-(4) above. Thus the sequence $\left\{K_{j}\right\}$ can be selected, and this concludes the proof of Theorem 2 .

From now on, $R$ will be an actual Dedekind ring, and we shall study $R$-modules. We begin by disposing of torsion modules. Call a torsion $R$-module primary (for the prime ideal $p$ ) if each of its elements is annihilated by a suitable power of $p$. It can be proved (in virtually the same way as for principal ideal rings) that every torsion $R$-module is uniquely a direct sum of primary modules.

Next let $R_{p}$ denote the quotient ring of $R$ relative to $p$, that is, the set of all elements $\alpha / \beta$ in the quotient field with $\beta$ prime to $p$. Then any $p$-primary $R$-module can as well be regarded as an $R_{p}$-module. Since $R_{p}$ is a principal ideal ring (indeed a discrete valuation ring), this makes the known theory of modules over principal ideal rings fully available. Consequently we have virtually nothing to say about torsion modules over a Dedekind ring; our interest is confined to. the torsion-free and mixed cases.

At the risk of possible ambiguity, we shall call an $R$-module decomposable if it is a direct sum of cyclic modules and finitely generated torsion-free modules of rank one. It is to be noted that when $R$ is a principal ideal ring, a decomposable module is precisely a direct sum of cyclic modules. A fundamental theorem of Steinitz [13] asserts that every finitely generated module over a Dedekind ring is decomposable $\left(^{3}\right)$. In Theorem 1, together with the remarks above concerning torsion modules, we have in effect given a new proof of this theorem.

We shall proceed to derive some results concerning decomposable modules.

Lemma 4. Let $R$ be a Dedekind ring, $M$ an $R$-module, $S$ a pure submodule, $x_{0}$ an element of $M / S$. Then there exists an element $x$ in $M$, mapping on $x_{0}$ mod $S$, and having the same order ideal as $x_{0}\left({ }^{4}\right)$.

Proof. The lemma is known if $R$ is a principal ideal ring, and the proof will consist of a reduction to that case. Let $I$ be the order ideal of $x_{0}$. If $I=0$, any choice of $x$ will do. So we suppose $I \neq 0$, let $\alpha$ be a nonzero element of $I$, and pick any $y$ in $M$ mapping on $x_{0}$. Then $\alpha y$ is in $S$ and, by the purity of $S$, there exists $s$ in $S$ with $\alpha y=\alpha s$. If $z=y-s$, then $\alpha z=0$, and $z$ (like $y$ ) maps on $x_{0} \bmod S$.

Now let $T$ be the torsion submodule of $M$. Then $S \cap T$ is pure in $T$. Moreover $T /(S \cap T)$ is in a natural way a submodule of $M / S$, and (in this sense) it contains $x_{0}$. The problem of lifting up $x_{0}$ has thus been reduced to the cor-

(3) A brief proof of this theorem is given by Chevalley in [4, Appendix 2].

${ }^{4}$ ) It is conversely true for a module $M$ over an arbitrary ring that purity of a submodule $S$ is implied by the ability to lift elements of $M / S$ with preservation of the order ideal. This stronger property should perhaps be used as the definition of purity when working over general rings. 
responding one for torsion modules, and it then reduces further to the primary case, since we can work separately in each primary case. This reduces the problem to the case of a principal ideal ring, and proves the lemma.

The next three theorems were proved for ordinary abelian groups by Kulikoff in [7, Theorem 1], [8, Theorem 2], and [7, Theorem 5]. His proofs extend without change to any principal ideal ring. We now provide the extension to Dedekind rings.

Theorem 3. Let $R$ be a Dedekind ring, $M$ an $R$-module, and $S$ a pure submodule such that $M / S$ is decomposable. Then $S$ is a direct summand of $M$.

Proof. In the light of Lemma 2, it suffices to prove this theorem in the cases where $M / S$ is itself cyclic or torsion-free of rank one. In the latter case the result follows from Lemma 3, and in the former case it is an immediate consequence of Lemma 4.

Theorem 4. Let $R$ be a Dedekind ring and $M$ a decomposable $R$-module. Then any submodule of $M$ is decomposable.

Proof. Case I. $M$ is a torsion module. We may work separately in each primary summand, and this reduces the problem to the case of modules over a principal ideal ring, where the theorem was proved by Kulikoff $[8$, Theorem 2].

Case II. $M$ is torsion-free. We may regard $M$ as the set of all wellordered vectors $\left\{\alpha_{\lambda}\right\}$, all but a finite number of coordinates zero, with $\alpha_{\lambda}$ ranging over an ideal $I_{\lambda}$ in $R$. Let $N$ be a submodule of $M$, and $N_{\lambda}$ the submodule of $N$ consisting of all elements whose coordinates all vanish after the $\lambda$ th. By mapping the general element of $N_{\lambda}$ onto its $\lambda$-coordinate we define a homomorphism of $N$ onto an ideal $J_{\lambda}$ contained in $I_{\lambda}$. (It may be that $J_{\lambda}=0$; this makes no difference.) By Lemma 3, the kernel of this homomorphism is a direct summand of $N_{\lambda}$. Let $S_{\lambda}$ be a complementary summand. Then each $S_{\lambda}$ is of rank one (or 0 ), and it is easy to see that $N$ is the direct sum of $\left\{S_{\lambda}\right\}$. Hence $N$ is decomposable.

Case III. $M$ is any decomposable module. Suppose $N$ is any submodule of $M$, and let $T$ be the torsion submodule of $M$. Then $N \cap T$ is the torsion submodule of $N$ and, by Case I, it is a direct sum of cyclic modules. Again $N /(N \cap T)$ may be regarded as a submodule of $M / T$ and is therefore decomposable by Case I. By Theorem 3 (or Lemma 3), $N \cap T$ is a direct summand of $N$. Hence $N$ is decomposable.

Theorem 5. Let $R$ be a Dedekind ring, $M$ an $R$-module, and $S$ a pure submodule of bounded order (that is, $\gamma S=0$ for some nonzero $\gamma$ in $R$ ). Then $S$ is a direct summand of $M$.

Proof. We begin by noting that the theorem is essentially known when $M$ is finitely generated. For then the torsion submodule $T$ of $M$ is a direct sum- 
mand (Theorem 1 or Steinitz's classical theorem). Moreover $S$ is a direct summand of $T$; for this may be checked separately in each primary component, which means we are over a principal ideal ring where the theorem is known [7, Theorem 5].

Another result we shall need is the classical theorem of Prüfer [10] which asserts that a module of bounded order is a direct sum of cyclic modules; this extends at once from the principal ideal to the Dedekind case, by passing as usual to the primary components.

We turn to the proof of Theorem 5 . We note (by the purity of $S$ ) that $S \cap \gamma M=0$. Let $P=S+\gamma M$, and write $M^{*}=M / \gamma M, P^{*}=P / \gamma M$. We propose to prove that $P^{*}$ is pure in $M^{*}$. For this purpose, suppose that $x^{*}=\alpha y^{*}$, where $x^{*}$ is in $P^{*}, y^{*}$ in $M^{*}$. Choose representatives $x, y$ in $M$ for $x^{*}, y^{*}$; we may take $x$ to be in $S$. We have an equation

$$
x=\alpha y+\gamma z \text {. }
$$

Since $S$ is a direct sum of cyclic modules, $x$ may be embedded in a finitely generated direct summand $Q$ of $S$; and since $S$ is pure in $M, Q$ is likewise pure in $M$. Let $Z$ be the submodule generated by $Q, y$, and $z$. Then (as observed above for the finitely generated case) $Q$ is a direct summand of $Z$. Let $y_{1}, z_{1}$ be the $Q$-components of $y, z$ in this decomposition. Then (3) yields $x=\alpha y_{1}+\gamma z_{1}$. But $\gamma z_{1}=0$, since $z_{1}$ is in $S$. Hence $x=\alpha y_{1}$. Passing to $M^{*}$, we obtain $x^{*}=\alpha y_{1}^{*}$, where $y_{1}^{*}$ is in $P^{*}$. We have thus proved that $P^{*}$ is pure in $M^{*}$.

Next, by a second application of Prüfer's theorem, $M^{*} / P^{*}$ is a direct sum of cyclic modules. By Theorem $3, P^{*}$ is a direct summand of $M^{*}$, say $M^{*}$ $=P^{*}+L^{*}$. Let $L$ be the inverse image in $M$ of $L^{*}$. Then $S \cap L=S \cap P \cap L$ $=S \cap \gamma M=0 ;$ and $S+L=S+\gamma M+L=P+L=M$. Hence $M=S \oplus L$, as desired.

There is a corollary of Theorem 5 which is worth stating.

Corollary. Let $M$ be a module over a Dedekind ring. If the torsion submodule of $M$ is of bounded order, then it is a direct summand of $M$.

We next take up the question of divisible modules, as defined in $\$ 2$.

Theоrem 6. Let $R$ be a Dedekind ring, and $D$ a divisible $R$-module. (a) If $M$ is an $R$-module, $S$ a submodule of $M$, and $f$ a homomorphism of $S$ into $D$, then $f$ can be extended to all of $M$. (b) If $D$ is a divisible submodule of an $R$ module $E$, then $D$ is a direct summand of $E\left({ }^{5}\right)$.

Proof. Let us begin by observing that (b) follows from (a). For let $D$ be a divisible submodule of $E$. We let $f$ be the identity map of $D$ onto itself, and, on the authority of (a), we extend $f$ to $E$. If $K$ is the kernel of the

(5) Baer [2] has given a necessary and sufficient condition, applicable to any ring $R$, for an $R$-module to be a direct summand of every larger module. What we are doing in effect is to show that, for Dedekind rings, his condition is equivalent to the simpler one which we are using. 
extended map, we have $E=D \oplus K$.

We proceed to prove (a), and we take up the torsion and torsion-free cases separately.

Case I. $D$ is torsion-free. By a transfinite induction, it suffices to demonstrate the possibility of extending $f$ to a single element $x$. Let $I$ denote the set of $\alpha$ in $R$ with $\alpha x$ in $S$. If $I=0$, it is evident that $f(x)$ may be chosen arbitrarily in $D$. Otherwise take any $\gamma \neq 0$ in $I$, and choose $y$ in $D$ with $\gamma y=f(\gamma x)$. We define $f$ on $S+R x$ by $f(s+\alpha x)=f(s)+\alpha y$. To see that this is a consistent definition, we have to check that $f(\beta x)=\beta y$ holds for all $\beta$ in $I$. This follows since $D$ is torsion-free and

$$
\gamma[f(\beta x)-\beta y]=\gamma f(\beta x)-\beta f(\gamma x)=0 .
$$

(It is to be observed that Case I works when $R$ is any integral domain.)

Case II. $D$ is a torsion module. We define $I$ as above and again assume $I \neq 0$. Define further $J$ to be the subset of $I$ consisting of all $\alpha$ with $f(\alpha x)=0$. Since $D$ is a torsion module, $J \neq 0$. Modulo $J, I$ is therefore a principal ideal: that is, $I=J+(\gamma)$. We again choose $y$ in $D$ so as to satisfy $\gamma y=f(\gamma x)$, and extend $f$ as in Case I. The fact that $f(\beta x)=\beta y$ for all $\beta$ in $I$ now follows since $\beta$ is a multiple of $\gamma \bmod J$.

Case III. $D$ is any divisible $R$-module. The torsion submodule $T$ of $D$ is again divisible. Now for torsion modules we have proved part (a) of the theorem in Case II. Moreover, as we observed in the first paragraph of the proof, part (b) follows from part (a). In other words, we know that $T$ is a direct summand of $D$. We may now perform the extension of $f$ separately in each summand, by citing Cases I and II. This completes the proof of Theorem 6.

It is now easy to give a complete structure theory for a divisible module $M$ over a Dedekind ring $R$. For by Theorem $6, M$ is a direct sum of a torsion module and a torsion-free module. The latter is merely a vector space over the quotient field $K$ of $R$. The structure of the former is known from the principal ideal ring case. In order to state the result, we define a module of type $p^{\infty}$ : take the torsion $R$-module $K / R$ and split it into its primary parts; the summand for the prime ideal $p$ is called the module of type $p^{\infty}$. The following theorem summarizes the facts.

Theorem 7. Let $R$ be a Dedekind ring with quotient field $K$. Then any divisible $R$-module is the direct sum of a vector space over $K$ and modules of type $p^{\infty}$ for various prime ideals $p$.

The following theorem is also an immediate consequence of Theorem 6 . It shows that in the study of Dedekind modules we can stick to the case where there are no divisible submodules.

Theorem 8. Any module $M$ over a Dedekind ring possesses a unique largest divisible submodule $D ; M=D \oplus E$ where $E$ has no divisible submodules. 
Finally we take up the question of indecomposable $\left(^{6}\right)$ modules over a Dedekind ring, and we generalize [7, Theorem 7].

Theorem 9. Let $R$ be a Dedekind ring and $M$ an $R$-module which is not torsion-free. Then $M$ possesses a direct summand which is either of type $p^{\infty}$ or isomorphic to $R / p^{n}$ for some prime ideal $p$.

Proof. Take a primary summand $P$ of the torsion submodule of $T$. It is proved in [7] that $P$ has a direct summand $Z$ which is either of type $p^{\infty}$ or isomorphic to $R / p^{n}$. In the first case $Z$ is divisible and so is a direct summand of $M$ by Theorem 6 . In the second case $Z$ is pure in $M$ and of bounded order. and so is a direct summand of $M$ by Theorem 5 .

We state explicitly the following consequence of Theorem 9.

TheOREM 10. An indecomposable module over a Dedekind ring $R$ cannot be mixed, i.e. it is either torsion-free or torsion. In the latter case it is either of type $p^{\infty}$ or isomorphic to $R / p^{n}$ for some prime ideal $p$.

5. Valuation rings. In this paper we are not attempting to handle anything more general than integral domains. So a valuation ring is an integral domain $R$ such that for any $\alpha$ and $\beta$ in $R$, either $\alpha$ divides $\beta$ or $\beta$ divides $\alpha$.

Essential to our purpose is the concept of maximality of a valuation ring. However we do not need either the original definition in [9], or the characterization by pseudo-convergence in [5]. We shall instead use the characterization by systems of congruences in [12] $\left.{ }^{7}\right)$. The property we need may as well be taken as the definition. We also introduce at this point a weakened form of maximality that we shall need; it is identical with that used in the hypothesis of [6, Theorem 11.1].

Definitron. Let $R$ be a valuation ring with quotient field $K$. We say that $R$ is maximal if the following is true: whenever $\alpha_{r} \in K$ and (integral or fractional) ideals $I_{r}$ are such that the congruences $\left({ }^{8}\right)$

$$
x \equiv \alpha_{r}\left(\bmod I_{r}\right)
$$

can be solved in pairs, then there exists in $K$ a simultaneous solution of all the congruences. If this condition holds whenever the intersection of the ideals $I_{r}$ is nonzero, we shall say that $R$ is almost-maximal.

REMARK. It should be noted that when $R$ is a discrete valuation ring, maximality coincides with completeness, and almost-maximality is vacuous.

It is convenient also to define maximality of modules $\left({ }^{9}\right)$.

( $\left.{ }^{6}\right)$ A module is indecomposable if it cannot be written as a direct sum, except in the trivial way. This is not the negation of "decomposable" in the sense used above.

(7) The fact that the definition about to be given is equivalent to maximality can be seen by combining remarks 2 and 4 on pages 48 and 50 of [12].

(8) Here, and in (4), the subscript $r$ has range in an arbitrary set.

$\left({ }^{9}\right)$ There should be no confusion between this meaning of "maximal" and the possible connotation of "largest." 
Definition. Let $R$ be any ring, and $M$ an $R$-module. We say that $M$ is maximal if the following is true: whenever ideals $I_{r} \subset R$ and elements $s_{r} \in M$ are such that

$$
x \equiv s_{r}\left(\bmod I_{r} M\right)
$$

can be solved in pairs in $M$, then there exists in $M$ a simultaneous solution of all the congruences.

LeMma 5. If $R$ is a maximal valuation ring and $J$ an (integral or fractional) ideal in $R$, then $J$ is a maximal $R$-module. If $R$ is an almost-maximal valuation ring and $J$ a nonzero integral ideal in $R$, then $R / J$ is a maximal $R$-module. The direct sum of a finite number of maximal modules is maximal.

Proof. We shall prove the first statement, leaving the others to the reader. We have then elements $s_{r}$ in $J$ and ideals $I_{r} \subset R$ such that the congruences (4), with $M$ replaced by $J$, can be solved in pairs within $J$. By hypothesis there exists in the quotient field of $R$ a simultaneous solution $x$ of these congruences, and the fact that $x$ satisfies a single one of the congruences suffices to show that $x$ itself is in $J$.

Our main theorems will be proved by a suitable process of "lifting up" elements, like that employed in Lemmas 3 and 4 . The first such theorem is the following.

THEOREM 11. Let $R$ be a valuation ring, $M$ a torsion-free $R$-module, and $S$ a pure maximal submodule of $M$ such that $M / S$ is a direct sum of modules of rank one. Then $S$ is a direct summand of $M$.

Proof. As usual, Lemma 2 reduces the problem to the case where $M / S$ is itself of rank one. Let $x_{0}$ be any nonzero element in $M / S$. We must find an element $x$ in $M$ which maps on $x_{0}$ and has the following property: whenever $x_{0}$ is a multiple of $\alpha \in R$ in $M / S$ (that is, whenever $x_{0}=\alpha y_{0}$ with $y_{0}$ in $M / S$ ), then $x$ is likewise a multiple of $\alpha$ in $M$; for when such an element $x$ is found, we need only take the pure submodule it generates to get the desired complementary summand to $S$.

We first pick any element $x^{*}$ in $M$ mapping on $x$. If $x_{0}=\alpha_{r} t_{r}\left(\alpha_{r} \in R\right.$, $t_{r} \in M / S$ ), pick $u_{r}$ in $M$ mapping on $t_{r}$; then $s_{r}=x^{*}-\alpha_{r} u_{r} \in S$. We do this for every possible choice of $\alpha_{r}$ and consider the set of congruences

$$
y \equiv s_{r}\left(\bmod \alpha_{r} S\right)
$$

thus obtained. We note that any two of these congruences have a simultaneous solution. In fact, if $\alpha_{j}$ divides $\alpha_{i}$, then $s_{i}$ works for both $i$ and $j$; for

$$
s_{i}-s_{j}=\alpha_{i} u_{i}-\alpha_{j} u_{j}=\alpha_{j}\left(\alpha_{j}^{-1} \alpha_{i} u_{i}-u_{j}\right),
$$

and the quantity in parentheses lies in $S$ since $S$ is pure. Let $y$ denote a 
simultaneous solution in $S$ of the congruences (5). Then the element $x=x^{*}$ $-y$ meets our requirements.

THEOREM 12. Let $R$ be a maximal valuation ring and $M$ a torsion-free $R$-module of countable rank. Then $M$ is a direct sum of modules of rank one.

Proof. We may exhibit $M$ as the union of an ascending sequence of pure submodules $S_{1} \subset S_{2} \subset \cdots$, where $S_{i}$ has rank $i$. Suppose by induction that we know that $S_{i}$ is a direct sum of modules of rank one. Then by Lemma 5 , $S_{i}$ is maximal. Also $S_{i+1} / S_{i}$ has rank one. Hence Theorem 11 is applicable and shows that $S_{i}$ is a direct summand of $S_{i+1}$. This in turn shows us that $S_{i+1}$ is a direct sum of modules of rank one. Hence $M$ is likewise a direct sum of modules of rank one.

The following consequence of Theorems 11 and 12 is worth stating.

THEOREM 13. Let $R$ be a maximal valuation ring, $M$ a torsion-free $R$-module of countable rank, $S$ a pure submodule of finite rank. Then $S$ is a direct summand of $M$.

Proof. The module $M / S$ is again torsion-free and again has countable rank; by Theorem 12, it is a direct sum of modules of rank one. Again, by Theorem 12, $S$ is the direct sum of a finite number of modules of rank one, hence maximal by Lemma 5 , hence a direct summand of $M$ by Theorem 11 .

REMARKs. 1. In the special case where $R$ is a complete discrete valuation ring, Theorem 12 may be stated as follows: any countably generated torsionfree $R$-module is the direct sum of a divisible module and a free module. This Theorem is implicit in the work of Prüfer [11].

2. The assumption of countable rank in Theorem 12 cannot be dropped. A counter-example is given by Baer in [1, Theorem 12.4]. Indeed let $R$ be any principal ideal ring (not a field) and $M$ the complete direct sum of an infinite number of copies of $R$. Then if $M$ (as an $R$-module) were the direct sum of modules of rank one, it would have to be free. But Baer proves that $M$ is not free.

3. In Theorem 13 we cannot allow $S$ to be of infinite rank. For example, let $R$ be any principal ideal ring (not a field), and $M$ a free $R$-module of infinite rank. Then we know that $M$ can be mapped homomorphically onto the quotient field of $R$, say with kernel $S$. Then although $S$ is pure it cannot possibly be a direct summand of $M$.

4. On the other hand, I have been unable to determine whether Theorem 13 still holds if $M$ is of uncountable rank. Indeed it seems to be unknown whether a module of uncountable rank over a maximal valuation ring (say the $p$-adic integers) might even be indecomposable.

5. Theorem 12 may be supplemented by a uniqueness theorem which is easily proved by the methods used by Baer in proving [1, Corollary 2.9]: let $R$ be any valuation ring and $M$ an $R$-module which is a direct sum of 
modules of rank one; then the expression is unique up to isomorphism.

Our final theorem will deal with torsion modules, and in preparation for it we prove a lemma.

LEмма 6. Let $R$ be an almost-maximal valuation ring, $M$ an $R$-module, $S$ a pure cyclic submodule with a nonzero order ideal, and suppose that $M / S$ is a direct sum of cyclic modules. Then $S$ is a direct summand of $M$.

Proof. It suffices (Lemma 2) to handle the case where $M / S$ is cyclic. Let $t_{0}$ be a generator of $M / S$, and $H$ the order ideal. Our problem is to find in $M$ an element $t$ mapping on $t_{0}$ and again having order ideal $H$, for then the cyclic submodule generated by $t$ will be a complementary summand to $S$. First pick any element $y$ in $M$ mapping on $t_{0}$. For any $\alpha_{r} \in H$ we have $\alpha_{r} y \in S$. Since $S$ is pure, $\alpha_{r} y=\alpha_{r} s_{r}$ with $s_{r}$ in $S$. Let $J$ be the order ideal of $S$, and let $K_{r}$ denote the ideal in $R$ consisting of all $\beta$ with $\beta \alpha_{r} \in J$; note that $K_{r} S$ is precisely the submodule of $S$ annihilated by $\alpha_{r}$. We consider the system of congruences thus obtained for all choices of $\alpha_{r}$ in $H$ :

$$
x \equiv s_{r}\left(\bmod K_{r} S\right),
$$

and observe that any two have a simultaneous solution; for if $\alpha_{j}$ divides $\alpha_{i}$, then

$$
\begin{aligned}
\alpha_{i}\left(s_{i}-s_{j}\right) & =\alpha_{i} s_{i}-\left(\alpha_{i} \alpha_{j}^{-1}\right) \alpha_{j} s_{j} \\
& =\alpha_{i} y-\left(\alpha_{i} \alpha_{j}^{-1}\right) \alpha_{j} y=0
\end{aligned}
$$

whence

$$
s_{j} \equiv s_{i}\left(\bmod K_{i} S\right),
$$

that is to say, $s_{j}$ works for both the $i$ th and $j$ th congruences. Now by Lemma $5, S=R / J$ is a maximal module. Hence the congruences (6) have a simultaneous solution $x$ in $S$. The element $t=y-x$ then has the desired properties: it maps on $t_{0}$ in $M / S$, and it has the order ideal $H$.

THEOREM 14. Any finitely generated module over an almost-maximal valuation ring is a direct sum of cyclic modules.

Proof. Let $M$ be the module. We first apply Theorem 1, and learn that the torsion submodule $T$ of $M$ is a direct summand, and that $M / T$ is free $\left({ }^{10}\right)$. The problem is consequently reduced to the torsion case, and we accordingly assume that $M$ is a torsion module.

Let $z_{1}, \cdots, z_{n}$ be a set of generators for $M$. Suppose that of these $n$ elements, $z_{1}$ has the smallest order ideal. This implies that the cyclic submodule $S$ generated by $z_{1}$ is pure. By induction on $n$, we may assume that

(10) One should of course observe that in a valuation ring all finitely generated ideals are principal, and hence invertible; thus the theorem is applicable. 
$M / S$ is a direct sum of cyclic modules. By Lemma $6, S$ is a direct summand of $M$. Hence $M$ is a direct sum of cyclic modules.

\section{BIBLIOGRAPHY} 68-122.

1. R. Baer, Abelian groups without elements of finite order, Duke Math. J. vol. 3 (1937) pp.

2. - Abelian groups that are direct summands of every containing abelian group, Bull. Amer. Math. Soc. vol. 46 (1940) pp. 800-806.

3. J. Braconnier, Sur les groupes topologiques localements compacts, J. Math. Pures Appl. vol. 27 (1948) pp. 1-85.

4. C. Chevalley, L'arithmétique dans les algèbres de matrices, Actualités Scientifiques et Industrielles, no. 323, Paris, 1936.

5. I. Kaplansky, Maximal fields with valuations, Duke Math. J. vol. 9 (1942) pp. 303-321.

6. - Elementary divisors and modules, Trans. Amer. Math. Soc. vol. 66 (1949) pp. $464-491$.

7. L. Kulikoff, Zur Theorie der abelschen Gruppen von beliebiger Mächtigkeit, Mat. Sbornik vol. 9 (1941) pp. 165-181 (Russian with Germany summary).

8. - On the theory of abelian groups of arbitrary power, Mat. Sbornik vol. 16 (1945) pp. 129-162 (Russian with English summary).

9. W. Krull, Allgemeine Bewertungstheorie, Journal für Mathematik vol. 167 (1932) pp. 160-196.

10. H. Prüfer, Untersuchungen über die Zerlegbarkeit der abzählbaren primären abelschen Gruppen, Math. Zeit. vol. 17 (1923) pp. 35-61.

11. - Theorie der abelschen Gruppen. II. Ideale Gruppen, Math. Zeit. vol. 22 (1925) pp. 222-249.

12. O. F. G. Schilling, Valuation theory, Mathematical Surveys, no. 4, New York, American Mathematical Society, 1950.

13. E. Steinitz, Rechtickige Systeme und Moduln in algebraischen Zahlkörpern, Math. Ann. vol. 71 (1912) pp. 328-354.

University of Chicago,

ChicAgo, Ill. 